\title{
Dual-Isotope Spectrometric Gamma Densitometer for Diagnostics of Three-Phase Oil-Water-Gas Flows
}

\author{
B. N. Sveshnikov ${ }^{a, *}$, S. N. Smirnov ${ }^{b, * *}$, A. Yu. Filippov ${ }^{c, * * *}$, and Yu. P. Filippov ${ }^{a}$ * \\ ${ }^{a}$ Joint Institute for Nuclear Research, Dubna, Moscow oblast, 141980 Russia \\ ${ }^{b}$ Institute of Physical and Technical Problems, Dubna, Moscow oblast, 141980 Russia \\ ${ }^{c}$ Moscow Power Engineering Institute (National Research University), Moscow, 111250 Russia \\ *e-mail:fyp@dubna.ru \\ **e-mail: orp@iftp.ru \\ ***e-mail:forcs2231@gmail.com
}

Received August 28, 2020; revised September 2, 2020; accepted September 2, 2020

\begin{abstract}
The operation of a gamma densitometer (GD) of ID $=100 \mathrm{~mm}$ is considered; it is intended for the diagnostics of three-phase oil-water-gas flows using the separationless method, when the composition of components is determined by a spectrometric analysis of gamma irradiation with two different photon energies. The ${ }^{241} \mathrm{Am}$ and ${ }^{137} \mathrm{Cs}$ isotopes, installed in the same housing, are used as gamma sources, while a gamma detector is made on the basis of the BGO crystal of $40 \mathrm{~mm}$ in diameter and height. Information about the design, measuring electronics, and main characteristics of the GD is given. The method used for the temperature stabilization of the recorded radiation intensities for both isotopes within a range of ambient temperature from 10 to $35^{\circ} \mathrm{C}$ is presented.
\end{abstract}

Keywords: separationless flowmeter, three-phase flow, two-phase flow, gamma densitometer, narrowing device, oil, water, gas

DOI: $10.1134 / \mathrm{S} 1547477121010118$

\section{INTRODUCTION}

For monitoring a mass flowrate of raw oil in the oil-extracting industry, together with large separation group measuring stations [1], compact separationless three-phase oil-water-gas flowmeters find growing application. The operating principle of these diagnostics systems, which are based, e.g., on a combination of a narrowing device (ND) and a gamma densitometer (GD), operating with the application of gamma sources with two different energies, is presented in [2$4]$. The composition of the three-phase flow components $\varphi, w$, and $\alpha$ is determined by three relations which are obtained on the basis of process descriptions, e.g., in [2] as functions of linear coefficients of absorption of gamma quanta with two different energies of photons in gas, water, and oil, as well as intensities of gamma rays (initial and passed through the flow) with two different energies, where $\varphi=A_{\mathrm{g}} /\left(A_{\mathrm{g}}+A_{\mathrm{l}}\right)$ is the actual volumetric gas content, $A$ is the cross-sectional area, the indices $\mathrm{g}$ and 1 refer to gas and liquid, $w=$ $\left(\rho-\rho_{o}\right) /\left(\rho_{w}-\rho_{o}\right)$ is the volumetric water cut, $\rho$ is the average density, the indices $w$ and o refer to water and oil, and $\alpha=(1-w-\varphi)$ is the volumetric oil content. To determine the volumetric flowrate of the mixture $Q$, a narrowing device is used, which operates in accordance with the fourth simplified relation with the horizontal orientation of the multiphase [5]: $\Delta P=\xi^{-2} \rho Q^{2}+\Delta P_{\mathrm{fr}}$, where $\Delta P$ is the measured full pressure drop on the narrowing device, which is composed of the drop in hydrodynamic pressure $\Delta P_{\mathrm{hd}}=$ $\xi^{-2} \rho Q^{2}$ due to the flow narrowing in the ND, estimated from the Bernoulli law under the assumption of the zero flow viscosity, and the frictional pressure drop is $\Delta P_{\mathrm{fr}} ; \xi=A_{1} A_{2}\left[2 /\left(A_{1}^{2}-A_{2}^{2}\right)\right]^{1 / 2}$ is the geometric parameter of the ND, and $A_{1}$ and $A_{2}$ are the larger and smaller cross sections of the conical ND. The influence of the latter component is more significant the higher the flow viscosity is, which is especially typical for oil, whose viscosity is several times more than that for water.

We recall the main principles of analyzing a component composition of multiphase flows. Therefore, the attenuation of parallel monochromatic beam of electromagnetic radiation is determined by the Lambert-Beer law [4]:

$$
I=I_{0} \exp (-\mu x),
$$

where $I_{0}$ is the initial intensity of radiation, $I$ is the intensity of the beam (covering distance $x$ through the substance), and $\mu$ is the linear coefficient of absorption. The mass absorption coefficient $\mu / \rho$ is frequently 
used, where $\rho$ is the substance density. A value of the mass absorption coefficient for mixtures and compounds can be found by the formula

$$
\mu / \rho=\Sigma w_{i}(\mu / \rho)_{i}
$$

where $w_{i}$ is the mass fraction of the $i$ th component and $(\mu / \rho)_{i}$ is the corresponding mass absorption coefficient. From (2), a value of the linear absorption coefficient can be obtained for mixtures and compounds:

$$
\mu=\Sigma \mu_{i} \alpha_{i},
$$

where $\alpha_{i}$ is the volumetric fraction of the $i$ th component and $\mu_{i}$ is the relevant mass absorption coefficient. Values of the mass absorption coefficient can be calculated theoretically or, e.g., borrowed from tables of National Institute of Standards and Technologies (United States).

In particular, for the two-phase water-gas mixture, the volumetric gas content of the flow $\beta=Q_{\mathrm{g}} /\left(Q_{\mathrm{g}}+Q_{w}\right)$ is related to the corresponding dimensionless GD characteristic $\beta_{\gamma}$,

$$
\beta_{\gamma}=1-\ln \left(I_{\mathrm{g}} / I\right) / \ln \left(I_{\mathrm{g}} / I_{\mathrm{w}}\right),
$$

cubic parabola, given, e.g., in [5], where $I$ i s the measured intensity of the passed gamma radiation, while the indices $g$ and $\mathrm{w}$ refer to gas and water and $Q$ is the volumetric flowrate.

While creating these GDs, a relation for error determination, e.g., the water cut of the oil-water mixture [6], serves useful purposes:

$$
\delta w= \pm \frac{1}{\mathrm{~d}\left(\mu_{0}-\mu_{\mathrm{w}}\right) \sqrt{t N^{*}}}
$$

where $d$ is the inner tube diameter; $\mu_{0}$ and $\mu_{\mathrm{w}}$ are the linear absorption coefficients for oil and water, respectively; $t$ is the time of measuring; and $N^{*}$ is the number of photons per unit time at the detector input. This relation is derived for quasi-stationary flows with stratified or annular flow regimes [6] under the condition that an error is determined only by a statistical error. From (5) it can be seen that the error $\delta w$ is inversely proportional to the flowmeter diameter and the square root of the source intensity and time of counting.

While creating experimental GDs for the diagnostics of three-phase oil-water-gas flows, the specifics of requirements for a measuring system should be taken into account. This system must ensure measuring temperatures $T$ of the flow and the flowmeter housing, flow pressure $P$, and pressure drop $\Delta P$ through the ND; it must respond to possible oscillations of parameters in flows with gas $[5,7]$ and average the measured parameters; it must implement a rather complex algorithm for processing the primary signals of the system for the purpose of obtaining the soughtafter quantities of the component composition and flowrates of three-phase flow components; and it must transmit information to a certain server using the standard data-exchange protocol. A system for measuring the ND signals $T, P$, and $\Delta P$ should be created on the basis of the industrial PC in the explosionproof casing, as is done, e.g., in [7]. The PC processor board is equipped with a flash disc; standard plugs for the TV monitor and keyboard connection; and Ethernet, USB, RS232, and RS485 interfaces. The electronics of the spectrometric GD with two gammaquanta energies should be compatible with the PC and should not overload its processor, the functions of which, as can be seen, are rather extensive.

The goal of this work was to search for or create a GD satisfying the abovementioned requirements, study its characteristics with filling by substances of different densities, and implement a method of temperature compensation of GD signals.

\section{MOCKUP OF THE EXPERIMENTAL GAMMA-DENSITOMETER PROTOTYPE}

In the available literature, a similar kind information of manufacturing companies, as a rule, is advertising; therefore, we had to create a diagnostics system for three-phase oil-water-gas flows all by ourselves. The mockup of this system is given in [7]. It is a combination of the conical ND (the larger and smaller diameters are 98 and $70 \mathrm{~mm}$ ) with the electronics module, based on the PC in explosion-proof housing, and the GD, mounted on a corrosion-resistant tube with an inner diameter of $98 \mathrm{~mm}$ according to Eq. (5). The ${ }^{241} \mathrm{Am}$ and ${ }^{137} \mathrm{Cs}$ gamma sources with activities of 450 and $5 \mathrm{mCi}$ and energies of 59.5 and $662 \mathrm{keV}$, respectively, are located in a single standard BGI-50P housing, whose design ensured a collimated beam. The BGI-50P housing is equipped with an "openclose" protective closure. For operability assurance of the lower energy gamma-quanta source, the diametrically opposite "windows" rated at the excessive pressure of $5 \mathrm{MPa}$ were fabricated according to the special technology. The block for radiation detection, specially created at the Institute of Physical and Technical Problems (IPTP) in the town of Dubna, was located opposite the BGI-50P housing. This block of detection with the so-called energy gates for both sources operated together with the laptop with which the signal spectra was processed, while their recorded integral characteristics (values of areas under energy peaks related to the time of accumulation of detector counts) were displayed and written into the memory in the form of readouts with dimensions of "count per second" (cps). The NaI crystal, which was intended for operation under laboratory conditions (the GET195-2011 multiphase-flow test bench, mentioned in [7]) was used as a scintillator in the block of detection. The GD layout during testing at the test bench is presented in Fig. 1. Because in the test-bench room, the temperature should change not frequently and not considerably, the temperature compensation of the $\mathrm{NaI}$ response whenever required was performed manually by chang- 


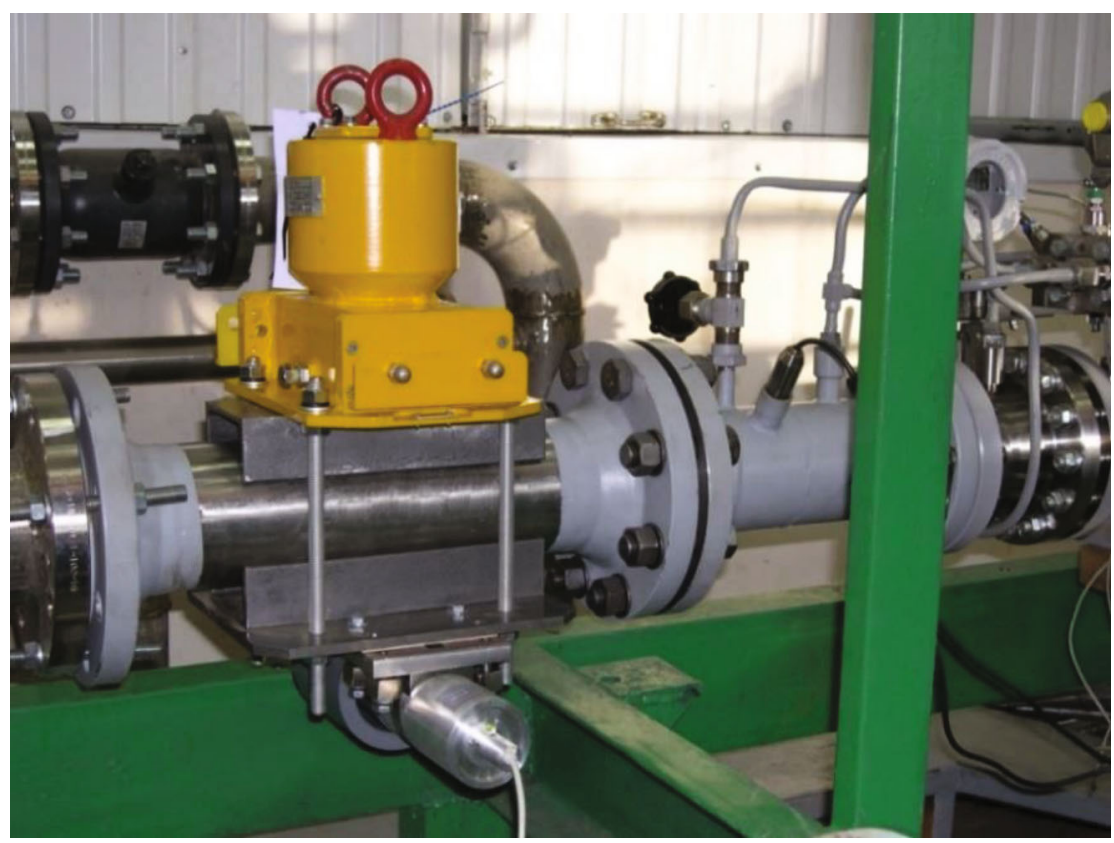

Fig. 1. External view of the mockup of spectrometric gamma densitometer during tests at the GET 195-2011 multiphase-flow test bench (All-Russian Research Institute for Flowmeter Survey, Kazan).

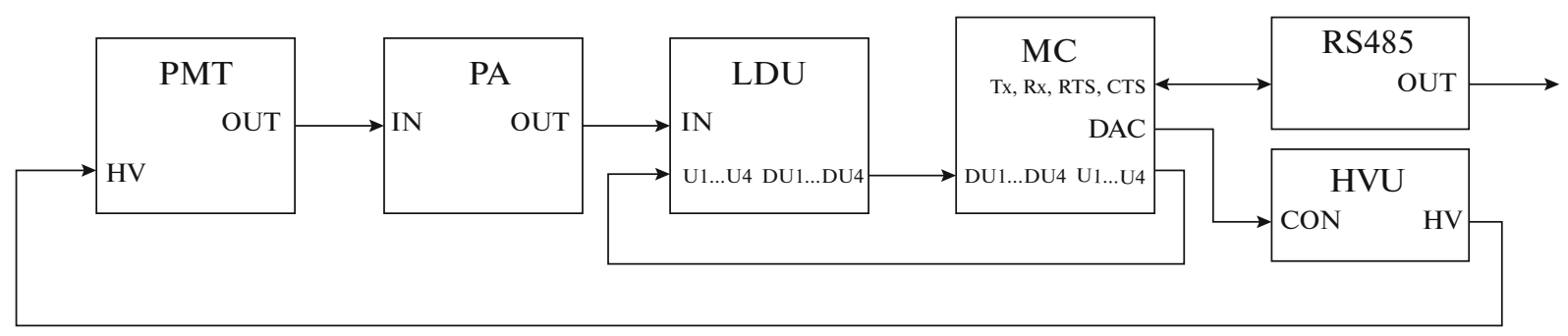

Fig. 2. Schematic diagram of the block of detection of the advanced spectrometric gamma densitometer: PMT is the photoelectric multiplier tube, PA is the preliminary amplifier, LDU is the level discriminator unit, MC is the STM32F100 microcontroller, RS485 is the galvanically isolated driver of the RS485 bus, HVU is the high voltage unit.

ing the energy gate position. Examples of successfully applying individual components of the GD $\left({ }^{241} \mathrm{Am}\right.$ or ${ }^{137} \mathrm{Cs}$ ) are presented in $[5,7]$ as aids for the diagnostics of two-phase flows of oil-stratal water, exxsol-water, water-air, and the liquefied natural gas (LNG). In particular, the system of diagnostics of two-phase oilsaltwater flows on the basis of the ${ }^{137} \mathrm{Cs}$ isotope and a conical ND satisfies the GOST requirements [8] within the $w$ range from 0 to 95\% [7].

The disadvantages of the GD mockup, which does not fully satisfy the above technical requirements, are obvious. In addition, the $\mathrm{NaI}$ crystal is hygroscopic [9], which requires a vibration-proof and sealed shell. In connection with this, the GD detector electronics was substantially improved at the IPTP.

\section{ADVANCED GAMMA DENSITOMETER}

In the advanced GD, the gamma source unit remained as before. In the block of detection, first and foremost, the $\mathrm{NaI}$ scintillator was replaced by a crystal of bismuth germanate $\mathrm{BGO}$ of $40 \mathrm{~mm}$ in diameter and height, which is nonhygroscopic and features higher radiation durability and substantially lower Compton scattering of ${ }^{137} \mathrm{Cs}$ gamma quanta. In addition, due to the high density and the larger effective atomic number, it predominates over sodium iodide by 1.27 times in efficiency of the total absorption-peak recording of gamma quanta, e.g., with an energy of $368 \mathrm{keV}$ with a slightly larger deexcitation time (by 1.2 times) [9].

The gamma-detector schematic diagram is presented in Fig. 2 [10]. The radiation from two sources interacts with the BGO crystal substance; this results in a light flash, which transforms to an electric pulse with the use of photoelectron multiplier tube (PMT). A signal from the PMT arrives at the input of the charge-sensitive preliminary amplifier (PA), where it is converted to a voltage pulse and filtered. From the PA output, the signal is supplied to inputs of four comparators of the level discriminator unit (LDU). At 


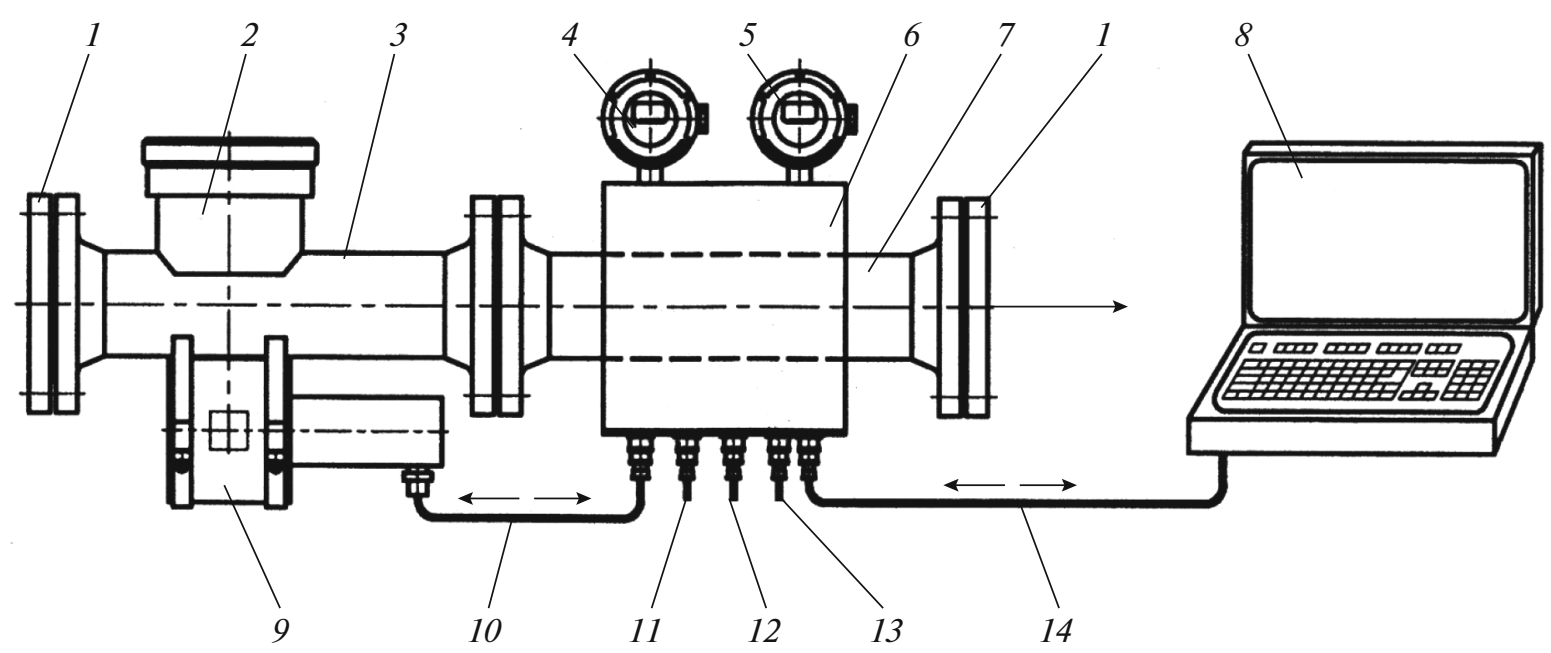

Fig. 3. Schematic diagram of electronics of the three-phase oil-water-gas flowmeter: (1) flange connector, (2) gamma source unit, (3) tube of $12 \mathrm{Kh} 18 \mathrm{~N} 9 \mathrm{~T}$ steel with an inner diameter of $98 \mathrm{~mm},(4)$ pressure transformer, (5) pressure drop transducer, (6) explosion-proof casing of the industrial PC, (7) narrowing device (ND), (8) notebook or server, (9) gamma block of detection, (10) cable of supply and data exchange through RS485, (11) connector of temperature sensors of the flow and the ND casing, (12) connector of ND pressure and pressure-drop sensors, (13) PC power supply of $24 \mathrm{~V} \mathrm{DC}$, and (14) Ethernet dataexchange cable over TCP/IP.

LDU inputs U1...U4, four pulse width modulated (PWM) signals from outputs of the microcontroller (MC) are supplied. In the LDU, the PWM signals are filtered and used as reference levels of comparators. The output pulses of comparators DU1...DU4 are delivered to counter inputs of four MC timers. Each counter timer of the MC counts the number of PA pulses exceeding the corresponding reference level which arrived during the measuring.

The counting results, in the form of 32-digit numbers, are stored in the MC memory after the measurement time termination. The analog signal, proportional to the PMT high voltage, is supplied to the CON input of HVU from the output of the digit-to-analog converter (DAC) of the MC. The measurement time, the reference levels, and high voltage value are software-defined from the RS485 channel and are saved by a particular channel command in the MC nonvolatile memory [10]. Through the RS485 cable, signals are transmitted to the processor board of the industrial $\mathrm{PC}$ in the form of counts in each channel.

The light contact between the PMT input window and the BGO crystal end is provided by means of a special lubricant and fixing holder. The temperature of the block of detection near the BGO is monitored by a temperature sensor arranged in the electronics board.

The block of detection ensures the gamma-radiation recording within an energy range from 50 to $1500 \mathrm{keV}$ and a range of equivalent dose powers from 0.09 to $50.0 \mu \mathrm{Sv} / \mathrm{h}$. The working range of ambient temperatures is from 5 to $35^{\circ} \mathrm{C}$. The spectrum data transfer from the block of detection to the PC processor board is implemented by means of the continuous trans- fer of entire spectrum buffer or by the page-by-page transfer. The continuous transfer is implemented for the spectra with sizes of 256, 512, and 1024 channels.

The schematic diagram of the three-phase flowmeter electronics is shown in Fig. 3. The power supply of block of detection 9 , the transfer of primary data of the ${ }^{241} \mathrm{Am}$ and ${ }^{137} \mathrm{Cs}$ spectra, and the temperature $T_{\mathrm{bd}}$ of the block of detection, as well as the data exchange, are implemented over cable 10 . The power supply of PC 6 is produced by the dc voltage of $24 \mathrm{~V}$. PC 6 includes the PCA-6763VG standard board with the AMD G-series T16R processor system with low consumption [11] and two domestically made boards for measuring the temperatures of the flow and the casing of narrowing device 7, as well as the pressure and pressure drop through the ND. If necessary, the PC may be equipped with additional standard boards for signal transmission, e.g., with a radio modem and with an eight-channel DAC for producing standard analog signals. The final treatment of spectra and the execution of the algorithm for processing all signals is performed by the PC processor board. The data exchange between PC 6 and server 8 is implemented over Ethernet cable 14 in accordance with the standard protocol TCP/IP. This configuration of electronics is intended for operation in heated rooms or special thermal chambers with temperature maintenance within the limits of $20 \pm 10^{\circ} \mathrm{C}$. Temperature $T_{\text {bd }}$ of the block of detection can be recorded every second or according to the specified algorithm.

The RS485 interface at the rate of $57600 \mathrm{kbit}$ per second is used as a communication link in the MC. 


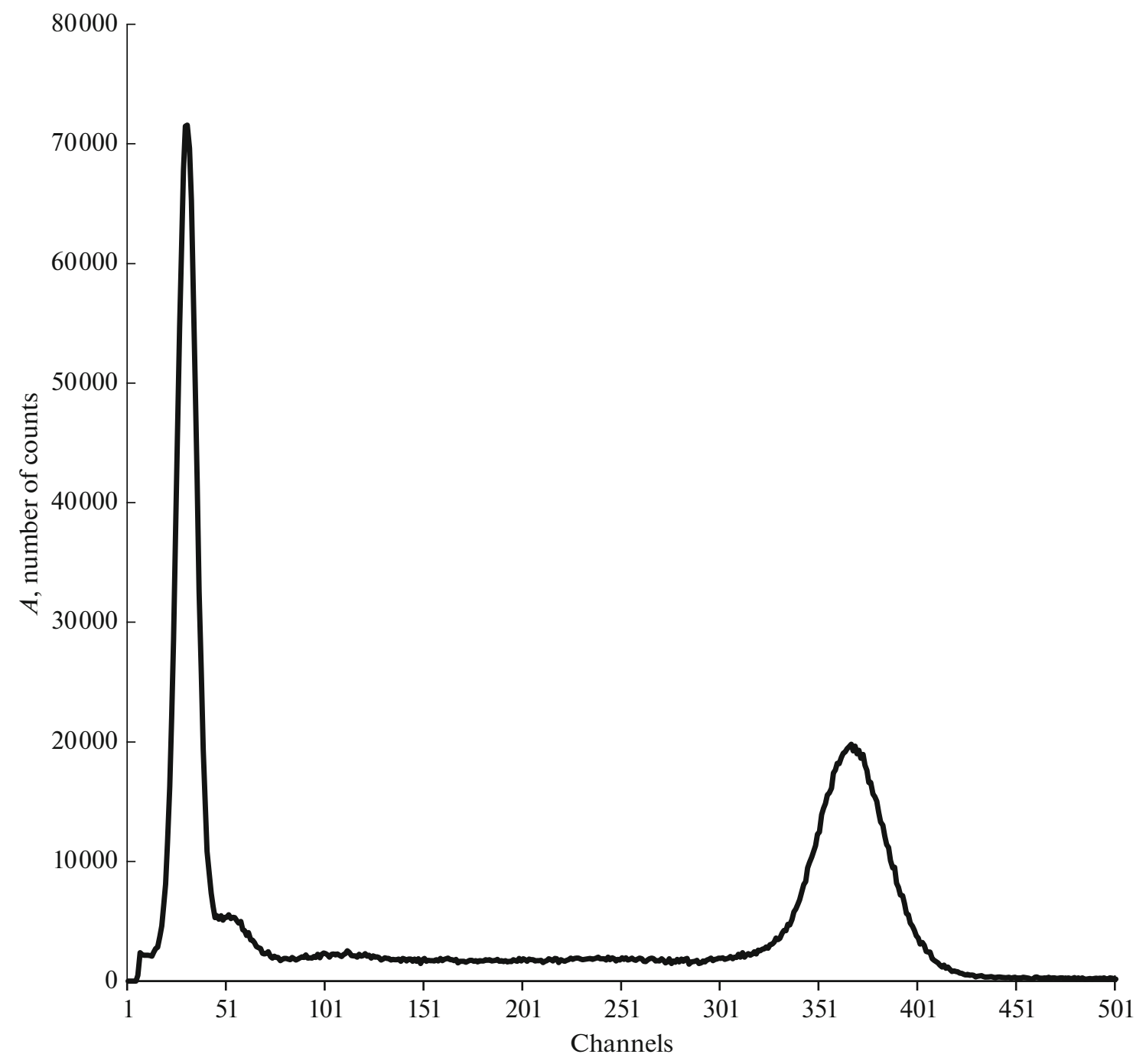

Fig. 4. Amplitude spectra of ${ }^{241} \mathrm{Am}$ and ${ }^{137} \mathrm{Cs}$ (peaks of full absorption of gamma quanta) with the atmospheric air inside at a temperature of $\sim 20^{\circ} \mathrm{C}$ and the data acquisition time of $60 \mathrm{~s}$.

The minimum time of data acquisition in the MC does not exceed $100 \mathrm{~ms}$. Since the volume of measured data is 4096 bytes ( 1024 four-byte words), then the measured data transmission from MC to PC takes the time of about $800 \mathrm{~ms}$. Thus, the minimum time of the system data acquisition (one timepoint) is no more than $1 \mathrm{~s}$. The software program in the PC through the communication channel presets the MC to the specified mode of operation and starts a measurement. During MC operation, signals from the temperature sensors and the sensors of pressure and pressure drop through the ND are measured. After the end of the measurement acquisition in the $\mathrm{MC}$, the software program in the $\mathrm{PC}$ reads the data received over the communication link closely, averages them out, calculates the sought-after quantities of the component composition and the flowrates of measured three-phase oil-watergas flow, and presents the measured and calculated quantities in textual and graphic forms.

\section{RESULTS}

As an example, the ${ }^{241} \mathrm{Am}$ and ${ }^{137} \mathrm{Cs}$ amplitude spectra with air inside the GD at the atmospheric pressure ( 1 bar) and a temperature of about $20^{\circ} \mathrm{C}$ are given in Fig. 4. The left edge of noises up to the fifth channel is removed by the lower level discriminator, and the signals here are not processed and not displayed on the spectrometer scale. From Fig. 4 it can be seen that the lower width of the ${ }^{241} \mathrm{Am}$ spectrum occupies approximately 45 channels (50-5) with an amplitude slightly larger than 70000 counts, while, for ${ }^{137} \mathrm{Cs}$, it is $\sim 125$ channels (425-300), respectively, with an amplitude of about 20000 counts. In this case, the areas under both energy peaks, classified as the dataacquisition time, are approximately identical; this will be shown below. The replacement of the substance inside the GD with the denser medium leads to the relevant decays of full absorption-peak amplitudes with 
Table 1. Absorption-peak amplitudes of ${ }^{241} \mathrm{Am}$ and ${ }^{137} \mathrm{Cs}$ (in counts) for different substances with an ambient temperature of about $20^{\circ} \mathrm{C}$ and data acquisition time in the detector of $60 \mathrm{~s}$

\begin{tabular}{l|c|c|c|c}
\hline \multicolumn{1}{c|}{ Substance } & Air, $P=1$ bar & Exxol D100 & Urban water & 10\% solution of NaCl in water \\
\hline${ }^{241} \mathrm{Am}$ & 71000 & 21300 & 16200 & 12730 \\
${ }^{137} \mathrm{Cs}$ & 19800 & 11500 & 10950 & 10410 \\
\hline
\end{tabular}

the persisting lower width of energy peaks. As an example, the absorption-peak amplitudes for different substances are presented in Table 1. However, the stabilities of reproduction of these amplitudes were insufficient for solving the stated problem.

For the further analysis of interaction of gamma quanta of two energies with different substances, the areas under full absorption peaks with regard to the background were used, classified as the time interval of data acquisition. The dimensionality of these characteristics is counts per second (cps). It should be noted that the background indicators on the left and on the right of peaks considerably differ both qualitatively and quantitatively. The peak data were used in which the corresponding derivatives are close to zero as boundaries of peaks in channels on the left and on the right. For example, for the data presented in Fig. 4, these boundaries on the left and on the right were within the limits of 15 and 50 for ${ }^{241} \mathrm{Am}$ and also 300 and 425 for ${ }^{137} \mathrm{Cs}$, respectively.

While working with BGO crystals, it should be taken into account that their light yield and fluorescent lifetime depend on temperature [9]. In this case, two regimes of GD operation should be distinguished: (i) the main regime, when the protective closure is open for the long-term operation and the thermal equilibrium is established with the heat removal from the block of detection to the environment, i.e., $T_{\mathrm{bd}}-T_{\mathrm{a}} \approx$ const, where $T_{\mathrm{bd}}$ and $T_{\mathrm{a}}$ are the temperatures of the block of detection and ambient air, respectively, and also the temperature correction of energy peaks is implemented, and (ii) the relatively short-term transient regime of operation, when $T_{\mathrm{bd}}$ rises, seeking an equilibrium without the temperature correction of peak positions.

We begin with the main regime of GD operation. Its characteristics at different temperatures were studied at the IPTP using a thermal chamber in which the air temperatures inside were recorded from 10 to $35^{\circ} \mathrm{C}$ with deviations within the limits of $\pm 0.5^{\circ} \mathrm{C}$. The GD characteristics depend not only on the BGO crystal temperature, but also on the substance inside the device, the density of which is also temperature dependent. In the ideal case, the temperature dependences of the recorded intensities for both isotopes would be preferably studied with the completely empty GD, i.e., with vacuum inside it. However, preliminary studies with air inside the GD under exces- sive pressure have shown that, in this case, a sensitivity is $150 \mathrm{cps} / \mathrm{bar}$ for both isotopes, which is equivalent to $\sim 125 \mathrm{cps} /\left(\mathrm{kg} / \mathrm{m}^{3}\right)$. The temperature variation from 10 to $35^{\circ} \mathrm{C}$ leads to a change in the air density by $\sim 0.1 \mathrm{~kg} / \mathrm{m}^{3}$, which is equivalent to a variation in the intensity $I$ approximately by $12-13 \mathrm{cps}$; this is, as is shown below, about two times less than the limiting absolute error of this variant of the instrument. In connection with this, the GD tests in the thermal chamber were conducted with the atmospheric air inside the GD. In addition, the natural heat transfer here is ensured in the GD elements.

The analysis of the influence of ambient temperature $T_{\mathrm{a}}$ on characteristics of full absorption peaks shows that the $T_{a}$ variation from 10 to $35^{\circ} \mathrm{C}$ is accompanied by the peak amplitude growth for ${ }^{241} \mathrm{Am}$ from $\sim 70000$ to $\sim 90000$ counts with a decrease in the halfwidth of the spectral line. In this case, the right boundary for ${ }^{241} \mathrm{Am}$ was compliant with the 54th channel $\left(B_{\mathrm{Am}}\left(10^{\circ} \mathrm{C}\right)=54\right)$, corresponding to the small peak of the spectrum, which is shown in Fig. 4. With the $T_{\mathrm{a}}$ growth, the peak displaced to the left and occupied the position $B_{\mathrm{Am}}\left(35^{\circ} \mathrm{C}\right)=39$ th channel, with an approximately identical width of boundaries in 35 channels in the background area. In its turn, for ${ }^{137} \mathrm{Cs}$ with the same $T_{\mathrm{a}}$ variation, the peak amplitude growth occurred from $\sim 19100$ to $\sim 25300$ counts, also with a decrease in the spectral-line halfwidth, while the left boundary of the peak displaced to the left rather considerably, from the $\sim 325$ th to the $\sim 225$ th channel, with the almost identical width of boundaries in 125 channels. As is shown below (Fig. 6), areas under the full absorption peaks are approximately identical for ${ }^{241} \mathrm{Am}$ and ${ }^{137} \mathrm{Cs}$ with a ratio of lower boundary widths of about $3.6(125 / 35)$ with about the same ratio of amplitudes $(70000 / 19100 \approx 3.7)$. In this case, for ${ }^{241} \mathrm{Am}$, the influence of the peak boundary position in the background region on the area under the peak can be neglected in the first approximation; moreover, the corresponding temperature dependences of gamma radiation were obtained, further taking the background into account under the assumption of the constant width of peak boundaries in 35 channels and with the fixed right boundary position $B_{\mathrm{Am}}=50$ th channel. It should be noted that background characteristics depend also on the density of the substance inside the GD. 


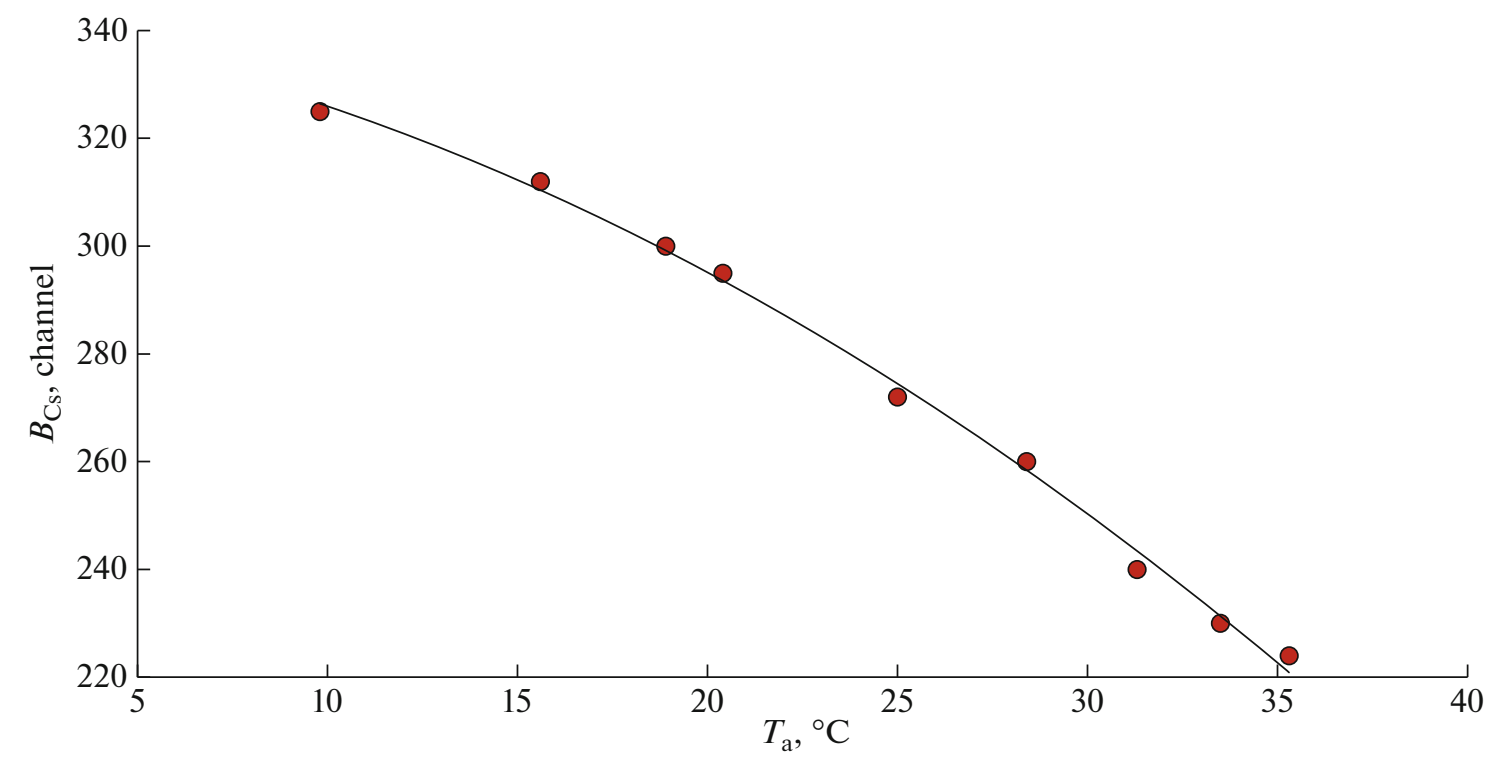

Fig. 5. Dependence of the left lower boundary $B_{\mathrm{Cs}}$ of the ${ }^{137} \mathrm{Cs}$ on the ambient temperature $T_{\mathrm{a}}$.

For ${ }^{137} \mathrm{Cs}$, a peak shape changes with the $T_{\text {bd }}$ rise in a more complicated manner, being accompanied, as was noted, by the amplitude increase and the narrowing of the spectral-line half-width; moreover, the background on the right almost is not recorded (Fig. 4), whereas, on the left, it grows as the temperature rises. In connection with this, a correction of the left boundary position $B_{\mathrm{Cs}}$ was made taking into account the background influence between peaks when the temperature $T_{\mathrm{bd}}$ changes. The configuration of this correction with the fixed width between the left and right peak boundaries, which is confirmed experimentally, is illustrated by Fig. 5. In connection with the fact that it is convenient to select the left boundary position $B_{\mathrm{Cs}}$ before the beginning of the operation, taking into account the ambient temperature, the correcting dependence is drawn in the form of $B_{\mathrm{Cs}}\left(T_{\mathrm{a}}\right)$, which is described by the quadratic parabola:

$$
B_{\mathrm{Cs}}=342.75-0.982 T_{\mathrm{a}}-0.07 T_{\mathrm{a}}^{2} .
$$

With allowance for the assumptions that were made, the test results are presented in Fig. 6, where each experimental point is obtained by averaging three series of measurements (by 10 measurements in a series with the time of $60 \mathrm{~s}$ of data acquisition in the detector) with six specified temperatures in the thermal chamber. It can be seen from this figure that both dependences $I\left(T_{\mathrm{bd}}\right)$ are linear functions with an almost identical slope of 59.5 and $60 \mathrm{cps} /{ }^{\circ} \mathrm{C}$ for ${ }^{241} \mathrm{Am}$ and ${ }^{137} \mathrm{Cs}$, respectively, with a maximum relative deviation of experimental points from approximating lines of $\pm 0.5 \%$. In this case, the intensity values of the transmitted radiation at the certain particular temperature, e.g., $T_{\mathrm{a}}=20^{\circ} \mathrm{C}$, at which, most likely, the GD static calibration is performed with two or three substances, depending on the problem to be solved, can be defined by the relation

$$
I=I_{\mathrm{m}} /\left(a T_{\mathrm{bd}}+b\right), \quad T_{\mathrm{bd}} \approx T_{\mathrm{a}}+20^{\circ} \mathrm{C},
$$

where $I_{\mathrm{m}}$ is the measured value of intensity of the transmitted radiation at the current temperature of the block of detection; $a$ and $b$ are the coefficients determined from the experimental data in Fig. 6. Within the investigated temperature range between $T_{\mathrm{bd}}$ and $T_{\mathrm{a}}$, an almost constant difference in the temperatures is established experimentally: $T_{\mathrm{bd}}-T_{\mathrm{a}} \approx 20^{\circ} \mathrm{C}$. Values of coefficients in Eq. (7) are $a=0.00367$ and $b=0.853$ for ${ }^{241} \mathrm{Am}$ and $a=0.00384$ and $b=0.847$ for ${ }^{137} \mathrm{Cs}$. In other words, the relative temperature coefficients are $a \approx 0.37 \% /{ }^{\circ} \mathrm{C}$ and $a \approx 0.38 \% /{ }^{\circ} \mathrm{C}$ for ${ }^{241} \mathrm{Am}$ and ${ }^{137} \mathrm{Cs}$, respectively.

If the temperature compensation using Eqs. (6) and (7) is activated, then the recorded radiation intensities $I_{\mathrm{Am}}$ and $I_{\mathrm{Cs}}$, reduced to the temperature $T_{\mathrm{a}}=$ $20^{\circ} \mathrm{C}$, are almost constant with a change in the ambient temperature and, consequently, in the temperature $T_{\text {bd }}$ and the instrument reacts only upon the composition of the substance inside it with the maximum relative deviations $\delta I / I_{\max } \approx \pm 0.22 \%$ in the air for both isotopes, which is confirmed by further experiments. It is very important for the practical application of similar devices.

In the transient regime, when equality $T_{\mathrm{bd}} \approx T_{\mathrm{a}}+$ $20^{\circ} \mathrm{C}$ is not reached yet in accordance with Eq. (7) and the mentioned temperature correction is not intro- 


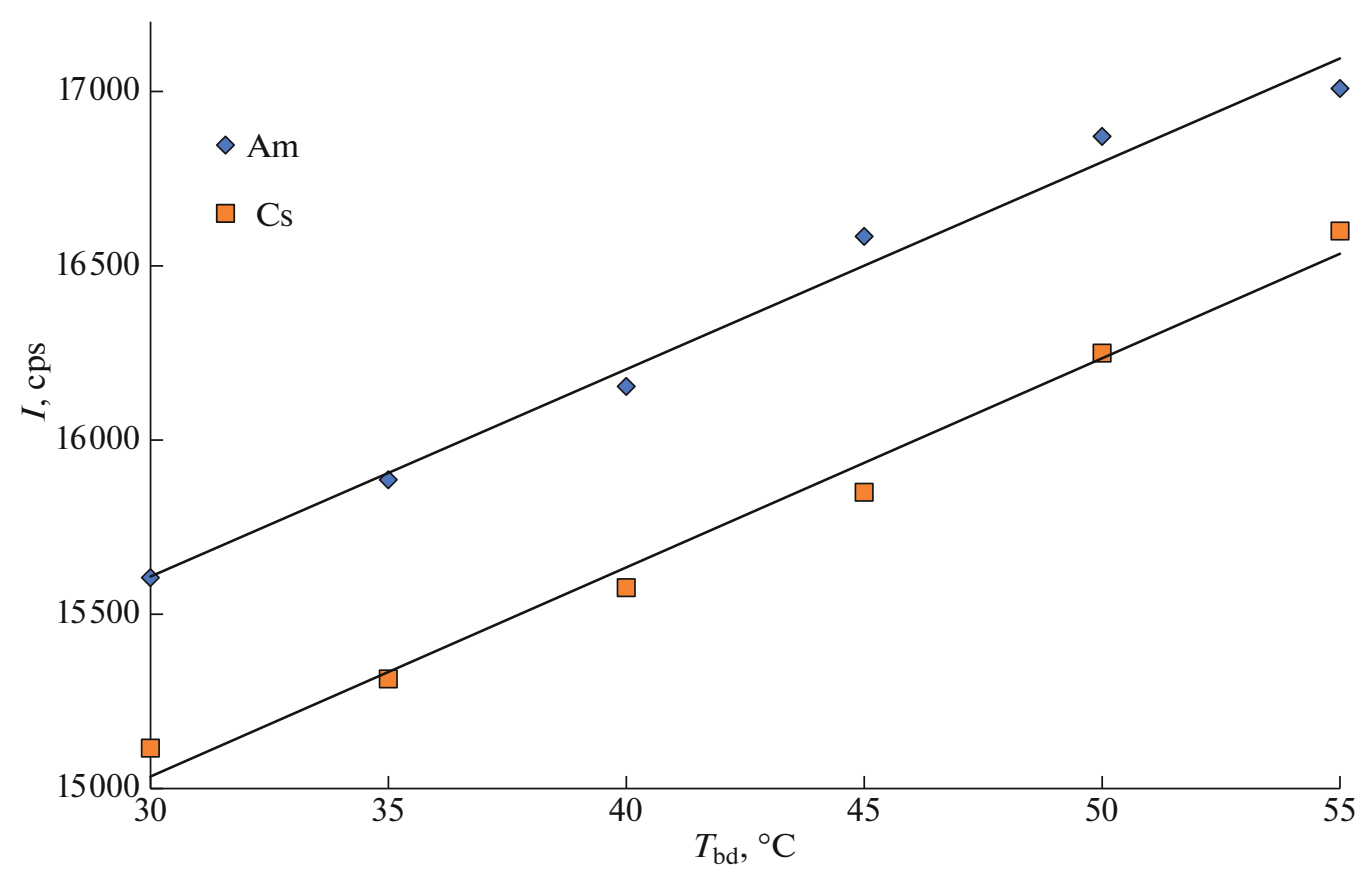

Fig. 6. Recorded gamma-radiation intensities $I$ [cps] as functions of the steady-state temperature of block of detection $T_{\mathrm{bd}}\left[{ }^{\circ} \mathrm{C}\right]$ for ${ }^{241} \mathrm{Am}$ and ${ }^{137} \mathrm{Cs}$ with air inside at the atmospheric pressure and the data acquisition time in the detector of $60 \mathrm{~s}$.

duced, the block of the detection has a specific feature, which is expressed in the fact that the intensity of the transmitted radiation for ${ }^{241} \mathrm{Am}$ grows with time, as is expected, due to the amplitude prevalence in the area of the spectrum under the peak in comparison with the decrease in the spectral-line halfwidth, whereas the intensity reduces for ${ }^{137} \mathrm{Cs}$, seeking stationary values. This is illustrated by Fig. 7, where the dynamics of temperature growth of the block of detection $T_{\mathrm{bd}}(t)$ is also shown. From Fig. 7 it can be seen that the stationary or main regime of operation begins approximately $3 \mathrm{~h}$ after the opening of the protective closure of the gamma-source unit. The unexpected (at first glance) behavior of the characteristic $I_{\mathrm{Cs}}(t)$ is explained by the opposite influence of two factors; in accordance with the first of which, the transmittedradiation intensity should grow due to an increase in the peak amplitude with the $T_{\mathrm{bd}}$ elevation. However, since the temperature correction is not introduced in this case, then, with the fixed position of the left boundary, the underestimation of counts of the left boundary of the full absorption peak occurs with the $T_{\mathrm{bd}}(t)$ growth and the relevant displacement of this peak to the left (Fig. 4). Since the quantitative contribution of the second factor is greater than that of the first factor, then the resulting influence is expressed by the reduction of the characteristic $I_{\mathrm{Cs}}(t)$, shown in Fig. 7b. The transient dynamical processes are described by quadratic parabolas (8) for ${ }^{241} \mathrm{Am}$ and (9) for ${ }^{137} \mathrm{Cs}$, as well as by logarithmic dependence (10) for $T_{\mathrm{bd}}(t)$ with maximum deviations $\delta T \approx \pm 0.1^{\circ} \mathrm{C}$ :

$$
\begin{gathered}
I_{\mathrm{Am}}=16053+0.861 t-0.0019 t^{2}, \\
I_{\mathrm{Cs}}=15686-1.442 t+0.0030 t^{2}, \\
T_{\mathrm{bd}}=36.6+0.6 \ln t .
\end{gathered}
$$

If the abovementioned specific feature of the block of detection is disregarded, then, during the transient period time, false results may be obtained, when the temperature dependences $I\left(T_{\mathrm{bd}}\right)$ for ${ }^{241} \mathrm{Am}$ and ${ }^{137} \mathrm{Cs}$ will have derivatives of opposite signs.

If it is necessary to reduce the statistical error of determining the flow component composition in accordance with (5), the design of the GD shown in Fig. 1 can be optimized by considerably decreasing the distance between the gamma-radiation source and detector, which allows the number of photons per unit time at the detector input $N^{*}$ to be increased approximately 2-3 times.

As to dependences of full absorption-peak amplitudes on temperature $T_{\mathrm{a}}$, they are described in the first approximation by the growing quadratic parabolas with values (in counts) of about $70000\left(15^{\circ} \mathrm{C}\right)$, $71000\left(20^{\circ} \mathrm{C}\right)$, and $79000\left(25^{\circ} \mathrm{C}\right)$ for ${ }^{241} \mathrm{Am}$ and $19200\left(15^{\circ} \mathrm{C}\right), 20000\left(20^{\circ} \mathrm{C}\right)$, and $22000\left(25^{\circ} \mathrm{C}\right)$ for

${ }^{137} \mathrm{Cs}$. In this case, deviations from the approximating dependences are appreciably larger than for character- 


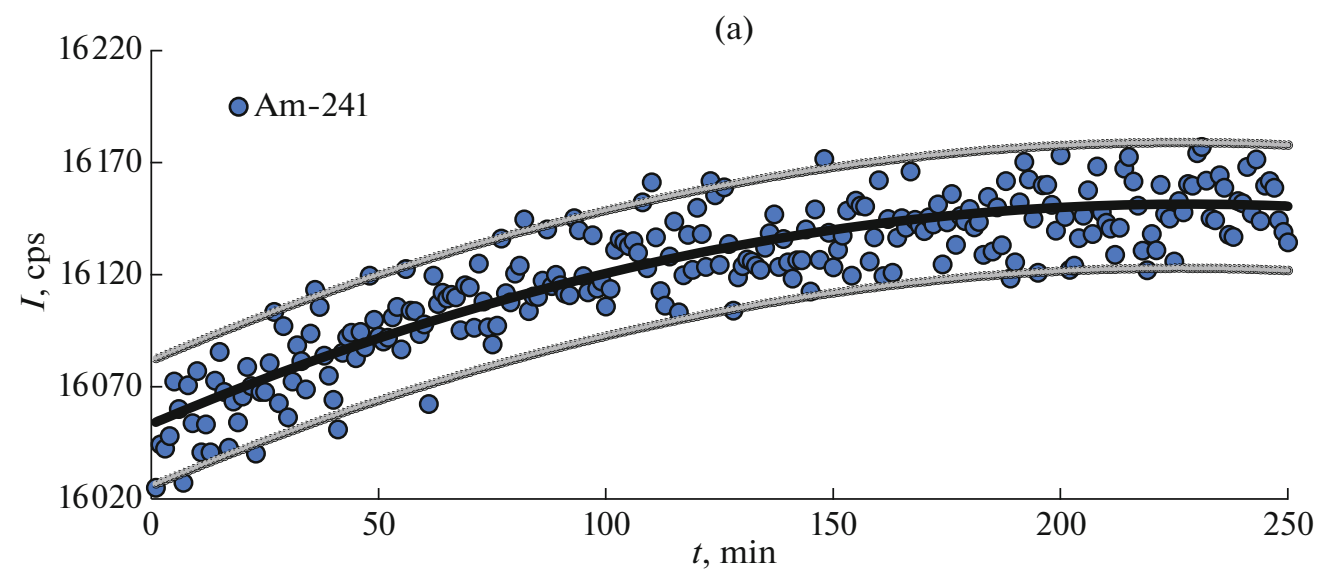

(b)

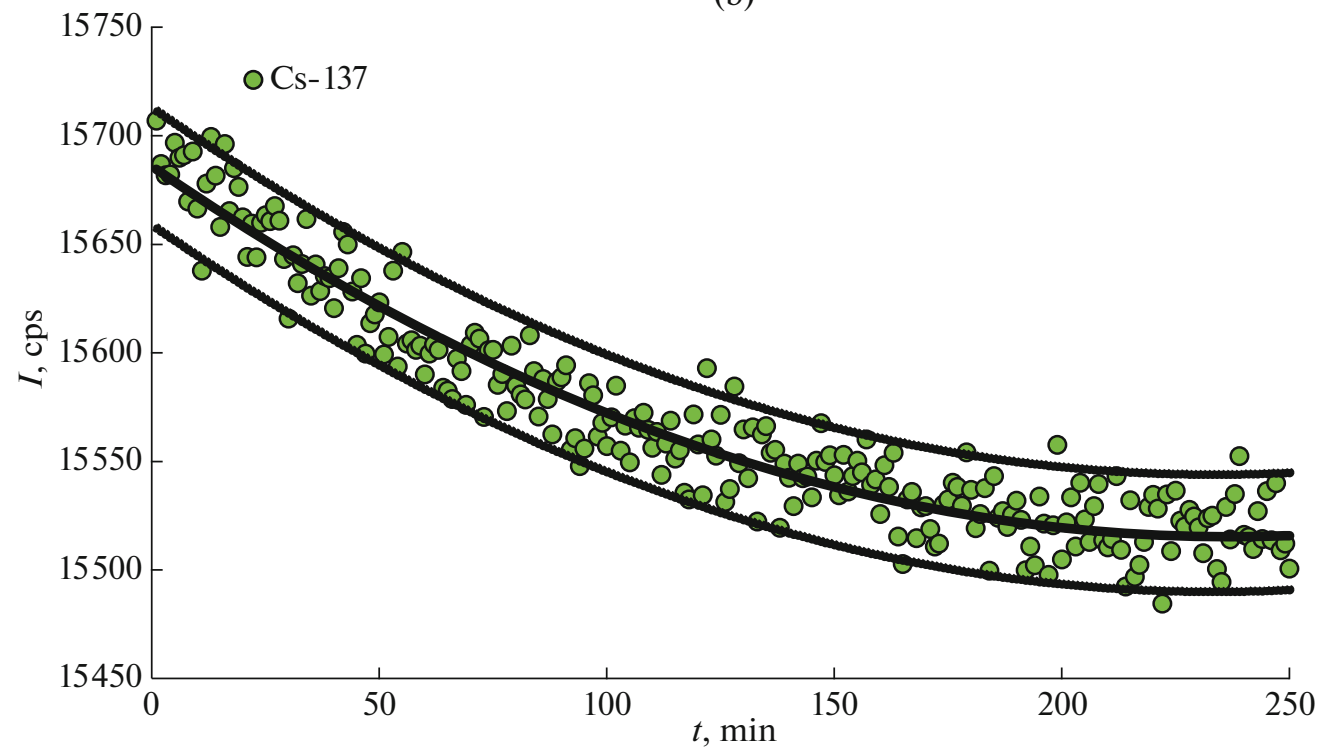

(c)

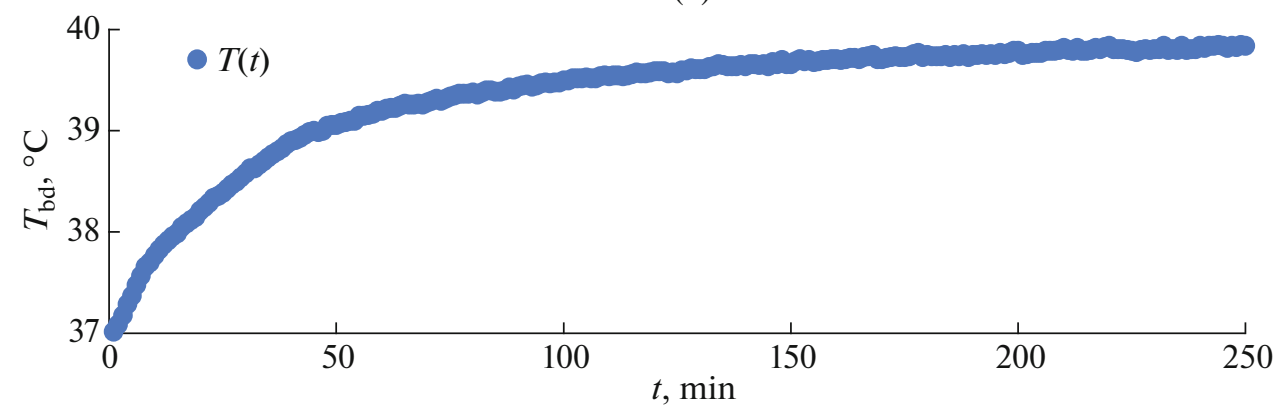

Fig. 7. Dynamics of recorded radiation intensities $I$ for (a) ${ }^{241} \mathrm{Am}$ and (b) ${ }^{137} \mathrm{Cs}$, as well as (c) temperatures $T_{\text {bd }}$ of the block of detection in air at the atmospheric pressure and ambient temperature $T_{\mathrm{a}} \approx 19^{\circ} \mathrm{C}$.

istics $I\left(T_{\mathrm{bd}}\right)$, presented in Fig. 6, which are used to solve the stated problem.

From the data presented in Fig. 7, the relative error of intensity measurement $\delta I / I$, which for the atmospheric air is $\sim \pm 0.17 \%$ for both isotopes, can be also estimated: $15525 \pm 27$ and $16150 \pm 28$ cps (average value \pm standard deviation). The marked boundaries of absolute deviations from generalizing dependences in Fig. 7 are derived for $95 \%$ of experimental points. In the presented measurements with the time of data acquisition in the detector of $60 \mathrm{~s}$, the contribution of statistical error can be neglected for each point, because, with times of 60 and $300 \mathrm{~s}$, the results are almost the same. The transfer to the denser substances 


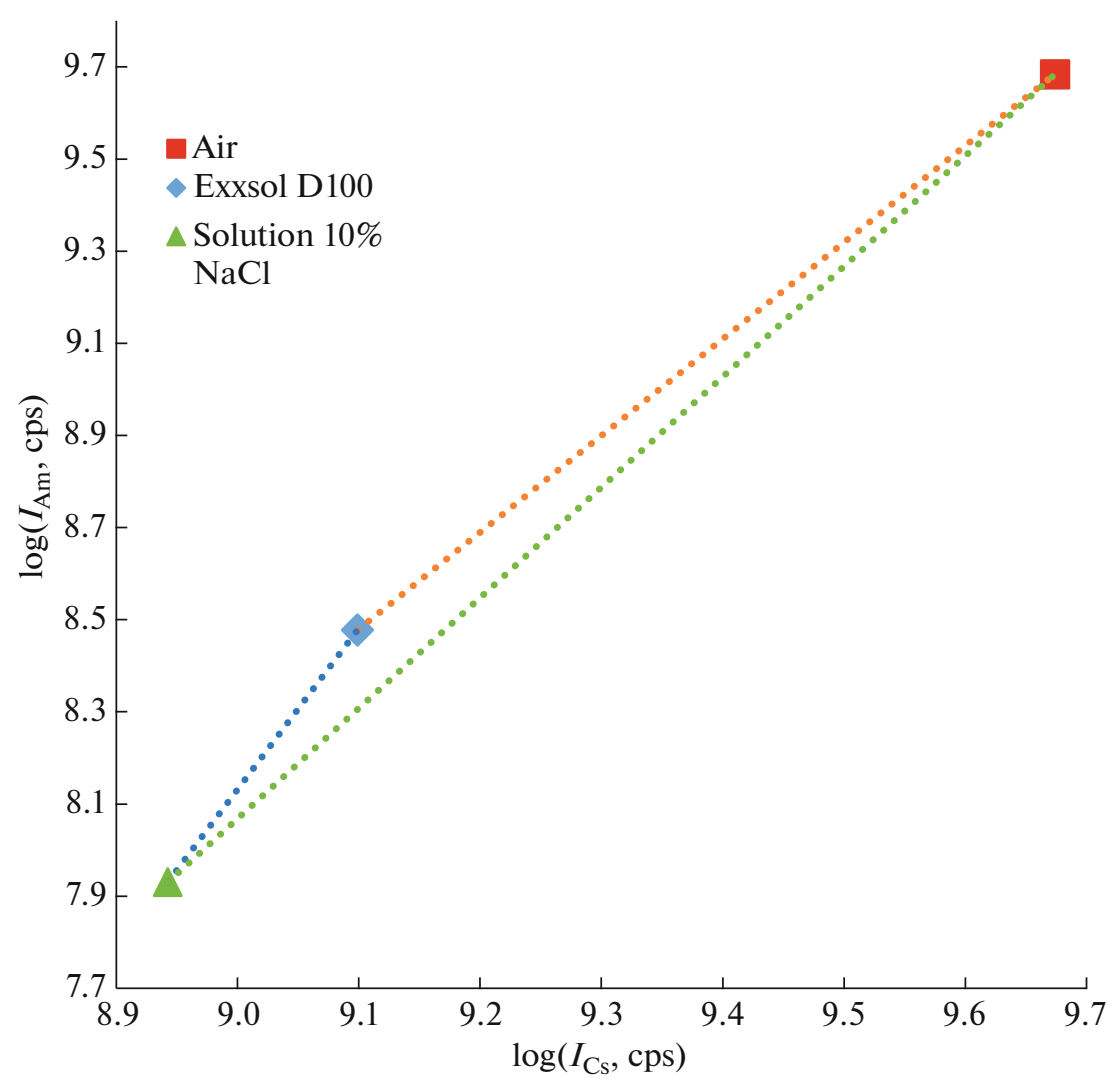

Fig. 8. Triangle of intensities of the transmitted radiation of the gamma densitometer for three-phase air-exxsol-salt water mixtures with the investigated ${ }^{241} \mathrm{Am}$ and ${ }^{137} \mathrm{Cs}$ isotopes.

is accompanied by some increase in relative errors, which, e.g., for Exxsol D100 with a density of $815.5 \mathrm{~kg} / \mathrm{m}^{3}$ (applied as an oil substitute at the test bench of the All-Russian Research Institute for Flowmeter Survey in Kazan [7]) are $\delta I / I_{\mathrm{Am}}=0.24 \%$ $(4240 \pm 10 \mathrm{cps})$ for ${ }^{241} \mathrm{Am}$ and $\delta I / I_{\mathrm{Cs}}=0.22 \%(9040 \pm$ $20 \mathrm{cps})$ for ${ }^{137} \mathrm{Cs}$. For water, these errors may grow up to $\delta I / I \approx 0.32 \%$.

For the analysis of characteristics of three-phase oil-water-gas mixtures, the so-called triangle of the component composition shown in Fig. 8 is of practical interest. Since the dependence of intensity of the radiation transmitted through the radiation flow under study is of exponential form in accordance with Eq. (1), then this characteristic is convenient to be presented in logarithmic coordinates. With an increase in the pressure of the flow under study, the gas point moves down in $\operatorname{Ln}(I)$ values. It can be seen from Fig. 8 that a triangle of intensities of transmitted radiation of the GD is comparatively narrow, and for successfully solving the problem of determining the component composition of three-phase mixtures, it is desirable to take further efforts for reaching the minimally possible values of $\delta I / I$. The technical capabilities of component-composition determination depend on the method for signal processing and on the quality of the initial data of the multiphase test bench, which requires further studies with the GD.

\section{CONCLUSIONS}

In this GD, created for the diagnostics of threephase oil-water-gas flows, the gamma source unit is made integrated (in one housing) on the basis of the ${ }^{241} \mathrm{Am}$ and ${ }^{137} \mathrm{Cs}$ isotopes, while the block of gammaquanta detection is based on the BGO crystal $40 \mathrm{~mm}$ in diameter and height, having a comparatively high radiation stability, relatively high efficiency of gammaradiation recording, and not having hygroscopicity. In comparison with the Vx analogue of the Schlumberger company [3], which operates with the ${ }^{133} \mathrm{Ba}$ isotope, this GD is distinguished by a much larger operational life due to the considerable difference in half-life time of ${ }^{133} \mathrm{Ba}$ and ${ }^{137} \mathrm{Cs}$ (approximately by three times).

To achieve a relatively high accuracy of measurements within the problem to be solved, it is expedient to use, as signals of the GD, the areas under peaks of recorded radiations, assigned to the pulse acquisition time, rather than the absorption-peak amplitudes. The relatively high stability of measuring intensities of the radiation transmitted through the flow under study 
for both isotopes is ensured by the developed method of temperature correction of signals of the spectrometric GD using linear relation (7), taking into account the assumptions made. This compensation is implemented by software in the processor board of the industrial computer of the created mockup of the separationless flowmeter. In this case, a position of the left boundary of the ${ }^{137} \mathrm{Cs}$ absorption peak is specified by quadratic dependence (6) on the ambient temperature or on the temperature of the block of detection with the fixed width of measurement channels, taking into account the background between energy peaks. If necessary, a similar procedure can be used for the ${ }^{241} \mathrm{Am}$ isotope to improve the measurement accuracy. Within the limits of assumptions made for this instrument, the correction of the right boundary of the ${ }^{241}$ Am peak may be neglected.

The relative errors of measured radiation intensities, obtained for both isotopes, $\delta I / I$ from $\sim 0.2$ to $\sim 0.35 \%$, which depend on the density of the analyzed substance inside the GD, are quite sufficient for solving most problems in two-phase flows of different component compositions and certain problems in the three-phase oil-water-gas flows. To extend the range of GD application in oil-water-gas flows, the measurement accuracy should be improved using all methods available.

\section{ACKNOWLEDGMENTS}

We thank Director of IPTP A.A. Smirnov highly skilled assistance in the creation of the spectrometric GD and helpful discussions.

\section{REFERENCES}

1. G. S. Abramov, "Analysis of metrological characteristics of units providing measuring of flow-rate parame- ters of oil wells' product,” Avtomatiz. Telemekh. Svyaz' Neft. Prom-sti, No. 1, 5-14 (2012).

2. I. M. M. Babelli, "Development of multiphase meter using gamma densitometer concept," in Proceedings of the International Nuclear Conference (1997), pp. 371389.

3. I. Atkinson et al., "New generation multiphase flowmeters from Schlumberger and Framo Engineering AS," in Proceedings of the 17th International Seminar on Flow Measurements in North Sea, Oslo, Norvegiya, 1999.

4. D. B. Bukur, J. G. Daly, and S. A. Patel, "Application of $\gamma$-ray attenuation for measurement of gas holdups and flow regime transitions in bubble columns," Ind. Eng. Chem. Res. 35, 70-80 (1996).

5. Yu. P. Filippov and A. Yu. Filippov, "Operation features of a narrowing device in separationless threephase flow-meter," Flow Meas. Instrum. 68, 101578 (2019).

6. L. Pan, "High pressure three-phase (gas/liquid/liquid) flow," PhD Thesis (Imperial College, London, 1996).

7. Yu. P. Filippov, I. D. Kakorin, A. M. Kovrizhnykh, and V. M. Miklayev, "Multiphase flow-meters for superconducting accelerators and others applications," Phys. Part. Nucl. Lett. 14, 602-614 (2017).

8. GOST (State Standard) No. R 8.615-2005. GSI. Measurement of the amount of oil and oil gas recovered from the depths. General metrological and technical requirements.

9. V. M. Bystritsky, E. V. Zubarev, A. V. Krasnoperov, S. Yu. Porohovoi, V. L. Rapatskii, Yu. N. Rogov, A. B. Sadovskii, A. V. Salamatin, R. A. Salmin, V. M. Slepnev, and E. I. Andreev, "Gamma detectors in explosives and narcotics detection systems," Phys. Part. Nucl. Lett. 10, 566 (2013).

10. Detection Unit BDGS-40.40, Prototype, User's Guide (Inst. Fiz.-Tekh. Probl., Dubna, 2017) [in Russian].

11. Prosoft-Sale of Industrial Computers. ADVANTECH Product Catalog. http://www.prosoft.ru.

Translated by M. Samokhina 